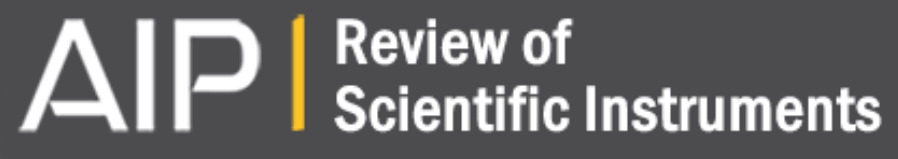

\section{A decade-spanning high-resolution asynchronous optical sampling terahertz time- domain and frequency comb spectrometer}

Jacob T. Good, Daniel B. Holland, Ian A. Finneran, P. Brandon Carroll, Matthew J. Kelley, and Geoffrey A. Blake

Citation: Review of Scientific Instruments 86, 103107 (2015); doi: 10.1063/1.4932567

View online: http://dx.doi.org/10.1063/1.4932567

View Table of Contents: http://scitation.aip.org/content/aip/journal/rsi/86/10?ver=pdfcov

Published by the AIP Publishing

\section{Articles you may be interested in}

Thin-sample limit for time-resolved terahertz spectroscopy

Appl. Phys. Lett. 97, 172110 (2010); 10.1063/1.3505340

Terahertz heterodyne spectrometer using a quantum cascade laser Appl. Phys. Lett. 97, 161105 (2010); 10.1063/1.3502479

Terahertz-range free-electron laser electron spin resonance spectroscopy: Techniques and applications in high magnetic fields

Rev. Sci. Instrum. 80, 073102 (2009); 10.1063/1.3155509

Ultrafast time-domain spectroscopy based on high-speed asynchronous optical sampling

Rev. Sci. Instrum. 78, 035107 (2007); 10.1063/1.2714048

Asynchronous optical sampling terahertz time-domain spectroscopy for ultrahigh spectral resolution and rapid data acquisition

Appl. Phys. Lett. 87, 061101 (2005); 10.1063/1.2008379

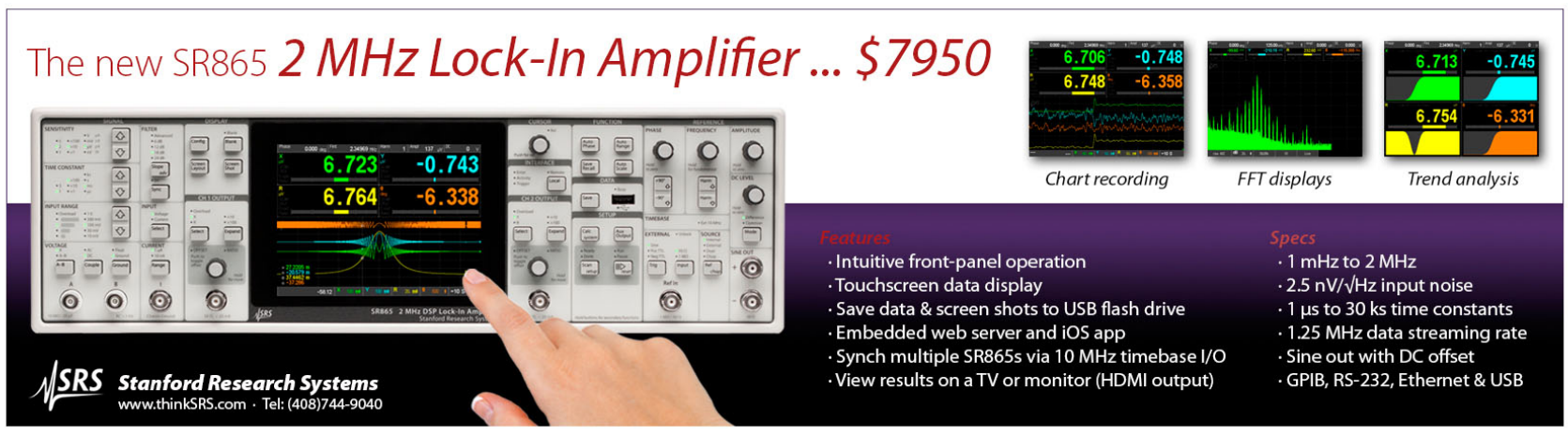




\title{
A decade-spanning high-resolution asynchronous optical sampling terahertz time-domain and frequency comb spectrometer
}

\author{
Jacob T. Good, ${ }^{1, a)}$ Daniel B. Holland, ${ }^{1,2, a)}$ Ian A. Finneran, ${ }^{1}$ P. Brandon Carroll, ${ }^{1}$ \\ Matthew J. Kelley, ${ }^{1,3}$ and Geoffrey A. Blake ${ }^{1,4}$ \\ ${ }^{1}$ Division of Chemistry and Chemical Engineering, California Institute of Technology, \\ Pasadena, California 91125, USA \\ ${ }^{2}$ Translational Imaging Center, University of Southern California, Los Angeles, California 90089, USA \\ ${ }^{3}$ Technology and Applications Center (TAC), Newport Corporation, Irvine, California 92606, USA \\ ${ }^{4}$ Division of Geological and Planetary Sciences, California Institute of Technology, \\ Pasadena, California 91125, USA
}

(Received 30 July 2015; accepted 24 September 2015; published online 14 October 2015)

\begin{abstract}
We present the design and capabilities of a high-resolution, decade-spanning ASynchronous OPtical Sampling (ASOPS)-based TeraHertz Time-Domain Spectroscopy (THz-TDS) instrument. Our system employs dual mode-locked femtosecond Ti:Sapphire oscillators with repetition rates offset locked at $100 \mathrm{~Hz}$ via a Phase-Locked Loop (PLL) operating at the 60th harmonic of the $\sim 80 \mathrm{MHz}$ oscillator repetition rates. The respective time delays of the individual laser pulses are scanned across a $12.5 \mathrm{~ns}$ window in a laboratory scan time of $10 \mathrm{~ms}$, supporting a time delay resolution as fine as $15.6 \mathrm{fs}$. The repetition rate of the pump oscillator is synchronized to a $\mathrm{Rb}$ frequency standard via a PLL operating at the 12th harmonic of the oscillator repetition rate, achieving milliHertz $(\mathrm{mHz})$ stability. We characterize the timing jitter of the system using an air-spaced etalon, an optical cross correlator, and the phase noise spectrum of the PLL. Spectroscopic applications of ASOPS-THz-TDS are demonstrated by measuring water vapor absorption lines from 0.55 to $3.35 \mathrm{THz}$ and acetonitrile absorption lines from 0.13 to $1.39 \mathrm{THz}$ in a short pathlength gas cell. With $70 \mathrm{~min}$ of data acquisition, a $50 \mathrm{~dB}$ signal-to-noise ratio is achieved. The achieved root-mean-square deviation is $14.6 \mathrm{MHz}$, with a mean deviation of $11.6 \mathrm{MHz}$, for the measured water line center frequencies as compared to the JPL molecular spectroscopy database. Further, with the same instrument and data acquisition hardware, we use the ability to control the repetition rate of the pump oscillator to enable $\mathrm{THz}$ frequency comb spectroscopy (THz-FCS). Here, a frequency comb with a tooth width of $5 \mathrm{MHz}$ is generated and used to fully resolve the pure rotational spectrum of acetonitrile with Doppler-limited precision. The oscillator repetition rate stability achieved by our PLL lock circuits enables sub-MHz tooth width generation, if desired. This instrument provides unprecedented decade-spanning, tunable resolution, from $80 \mathrm{MHz}$ down to sub-MHz, and heralds a new generation of gas-phase spectroscopic tools in the THz region. @ 2015 AIP Publishing LLC. [http://dx.doi.org/10.1063/1.4932567]
\end{abstract}

\section{INTRODUCTION}

Over the past three decades, terahertz time-domain spectroscopy (THz-TDS) has developed into a widely used technique that covers a key portion of the electromagnetic spectrum. ${ }^{1} \mathrm{THz}$ photons, with frequencies ranging from 0.1 to $10 \mathrm{THz}$, can interrogate a diverse suite of atomic and molecular degrees of freedom, including semiconductor phonon modes, ${ }^{2}$ the collective large-amplitude motions in proteins, ${ }^{3}$ hydrogen bonding in aqueous systems, ${ }^{4}$ the gas-phase rotational transitions of small molecules, ${ }^{5}$ and fine structure splittings in light atoms ${ }^{6}$ to name but a few examples. Such unique spectral signatures are particularly important to studies of the cold, dense clouds in the interstellar medium, and THz-TDS has the potential to provide high-quality measurements of the characteristic $\mathrm{THz}$ fingerprints of both gas-phase species ${ }^{7}$ and solid-phase molecular ices, ${ }^{8,9}$ and also more refractory

a) J. T. Good and D. B. Holland contributed equally to this work. materials that govern the assembly of stars and planetary systems.

Bandwidth covering the entire $\mathrm{THz}$ region is important for nearly all applications of THz-TDS, but gas-phase THz-TDS is particularly demanding as it additionally requires high frequency resolution (ideally Doppler-limited) across the entire bandwidth of interest. For THz-TDS to achieve such high resolution across intervals exceeding a decade in the frequencydomain requires sampling of long time delays with high temporal fidelity. This in turn requires a mechanism for generating an accurate time delay between $\mathrm{THz}$ generation and detection subsystems.

In a typical THz-TDS setup, ${ }^{10}$ a pulse train from an ultrafast mode-locked laser is split into two. The first pulse train is used as a pump to generate a $\mathrm{THz}$ pulse train, and the second pulse train is sent to a mechanical delay stage that adds an adjustable amount of path length corresponding to a variable time delay. This enables use of the second pulse train as a probe to sample the entire $\mathrm{THz}$ waveform, over the course of many THz pulses. Such a delay stage based 
THz-TDS system was recently demonstrated achieving an unprecedented bandwidth of $\sim 20 \mathrm{THz}$, but provided limited frequency resolution. ${ }^{11}$ While simple and robust, delay stages suffer from several limitations: moving parts present a challenge in maintaining uniform alignment and focusing of the probe pulse train throughout the scan, the scan rate is limited by the speed of motors, and the length of time delay scanned is limited by the length of the stage. These limitations make achieving both Doppler-limited resolution and decade spanning bandwidth extraordinarily challenging for conventional delay stages. Before turning to our specific implementation of a dual oscillator THz-TDS system, we first outline some prior approaches to overcoming the limitations imposed by delay stages.

\section{A. High-resolution THz spectroscopy}

The suite of THz-active processes outlined above yield an extraordinary range of dynamical time scales and spectroscopic signatures. Accordingly, of the advances in the generation and detection of $\mathrm{THz}$ radiation that have driven the development of a wealth of instrumentation and techniques for $\mathrm{THz}$ spectroscopy, some have specialized in bandwidth and others in resolution. An attractive approach to avoiding the difficulty of high-resolution time-domain sampling is to use a narrow linewidth $\mathrm{THz}$ source and sample directly in the frequency domain with a cryogenically cooled bolometer. A few examples of the application of this approach to gas-phase spectroscopy in the $\mathrm{THz}$ region are provided below.

Frequency multiplier submillimeter spectrometers (FMSSs), for example, continue their significant history of improvement, with recent extensions into the far-infrared, providing a sensitive method for acquiring very high resolution $\mathrm{THz}$ gas-phase spectra. ${ }^{12}$ Tunable continuous-wave (CW) quantum cascade lasers (QCLs) have also been used as a source of high brightness $\mathrm{THz}$ radiation, achieving sub-Doppler THz spectra of gas-phase samples. ${ }^{13}$ Recently, a static frequency comb spanning an octave from 1.64 to $3.35 \mathrm{THz}$ with a few $\mathrm{kHz}$ linewidth was generated by a QCL but interleaving of the comb to produce Doppler-limited spectra has not yet been demonstrated. ${ }^{14}$ The drawback of both QCL based THz spectrometers and FMSSs is their narrow bandwidth coverage due to the use of $\mathrm{CW} \mathrm{THz}$ sources. To obtain high precision measurements across multi-octave intervals requires prohibitively long scan times as the source must be tuned. This limitation has driven the development of methods for the high-resolution detection of $\mathrm{THz}$ pulses readily generated by ultrafast oscillator pulses.

\section{B. Asynchronous optical sampling based $\mathrm{THz}$ time-domain spectroscopy}

ASynchronous OPtical Sampling (ASOPS) is a technique that eliminates the need for a delay stage by employing two ultrafast mode-locked lasers with one as the pump and the other as the probe. ${ }^{15}$ Importantly, ASOPS techniques can be used in any region of the electromagnetic spectrum with processes driven by ultrafast laser pulses. The pump laser runs at a repetition rate $f_{\text {pump }}$ and the probe laser is offset locked to a slightly slower repetition rate $f_{\text {probe }}$, such that their repetition rate offset $\Delta f$ remains constant. That is, $\Delta f=f_{\text {pump }}-f_{\text {probe }}$.

In most systems, the time delay, $\tau_{d}$, covered is the inverse of the pump laser repetition rate $\left(\tau_{d}=1 / f_{\text {pump }}\right)$, since the ultrafast process of interest is initiated each time the pump laser fires. Periodically, a particular probe pulse will be delayed by exactly $\tau_{d}$ relative to a particular pump pulse, that is, the probe pulse will synchronize with the following pump pulse. The length of scanning time, $t_{d}$, needed to allow this pump-probe synchronization to occur is simply the inverse of the repetition rate offset, or $t_{d}=1 / \Delta f$. Scan rates from 100 to $1000 \mathrm{~Hz}$ are readily accessible via offset locking even for long time delays.

A theoretical maximum time-delay resolution, $\Delta \tau_{d}$, for an ASOPS system can be calculated by dividing the time delay, $\tau_{d}$, by the number of probe pulses, $N_{\text {probe }}$, available across the full time delay during each scan period, $t_{d}$, or $\Delta \tau_{d}$ $=\tau_{d} / N_{\text {probe }}$. The number of probe pulses available is given by the equation $N_{\text {probe }}=f_{\text {probe }} \times t_{d}$. Together, these equations yield the expression $\Delta \tau_{d}=\left(\tau_{d} / t_{d}\right) \times\left(1 / f_{\text {probe }}\right)$. The ability to access fine time-delay resolutions across long time delays makes ASOPS an attractive method for high-resolution timedomain spectroscopy.

The particular point in delay time, $\tau(t)$, that is sampled is a function of scan time elapsed between a particular probe pulse at time, $t$, and the time corresponding to the most recent overlap of pump and probe pulses, denoted by $t_{0}$. When the elapsed scan time $\left(t-t_{0}\right)=t_{d}$, a new scan begins with a new $t_{0}$. These relationships can be condensed into the equation $\tau(t)=\left(\tau_{d} / t_{d}\right) \times\left(t-t_{0}\right),{ }^{16}$ where the ratio $\tau_{d} / t_{d}$ is the time scaling factor introduced by ASOPS. Effectively, this scaling factor converts the ultrafast time scales associated with the laser pulses into a scan (laboratory wall clock) time that is far more amenable to sampling by conventional electronics, and once characterized allows the generation of a time delay axis directly from the scan time axis of the acquired data.

ASOPS may be applied to THz-TDS by using two ultrafast oscillators, one to pump a broadband $\mathrm{THz}$ emitter and the other to probe the generated $\mathrm{THz}$ radiation using detection in the time domain, typically via electro-optic (EO) sampling. ${ }^{17}$ Frequency content is extracted from the time-domain data by a fast Fourier transform (FFT) allowing THz-TDS to overcome broadband detector noise through the multiplex principle. To highlight the potential of ASOPS based THz-TDS, the capabilities of two recent ASOPS-THz-TDS instruments are listed here: a high-speed $1 \mathrm{GHz}$ oscillator ASOPS-THz-TDS system with $7 \mathrm{THz}$ of bandwidth that has achieved a mean frequency deviation of $142 \mathrm{MHz}$ when measuring the line centers of water vapor absorptions from 0.2 to $6.3 \mathrm{THz},{ }^{18,19}$ and a high-resolution ASOPS-THz-TDS system based on two Er-doped fiber lasers achieved a mean frequency deviation of $4 \mathrm{MHz}$ when measuring line centers of acetonitrile absorptions from $642.0 \mathrm{GHz}$ to $643.2 \mathrm{GHz} .{ }^{20}$ We stress that a single system has yet to demonstrate the ideal combination of high frequency resolution applicable to Doppler-limited spectroscopy while maintaining multi-decade spanning $\mathrm{THz}$ bandwidth. It should also be emphasized that the application of a given ASOPSTHz-TDS system to Doppler-limited spectroscopy is best accomplished through the operation of the system as a $\mathrm{THz}$ frequency comb spectrometer (THz-FCS), discussed next. 


\section{THz frequency comb spectroscopy}

The stabilization of the repetition rates of the Ti:Sapphire oscillators by PLL electronics ensures precision time delay sampling of a $\mathrm{THz}$ pulse train across a continuous time delay axis. Since the conversion of optical photons to mobile charge carriers within the $\mathrm{THz}$ emitter is sensitive to the intensity but not to the phase of the electric field of the pump laser optical pulse train, the resulting $\mathrm{THz}$ output is effectively CEP stabilized, with zero phase offset. This means that the instrument is always operating as a THz-FCS, with the frequency resolution determined by the length of continuous time delay sampled. ${ }^{21,22}$

In ASOPS-THz-TDS mode, most instruments sample a time delay of $1 / f_{\text {pump }}$ corresponding to the spacing between $\mathrm{THz}$ pulses in the time-domain. With proper data acquisition hardware, the width of the sampling window acquired, $N_{\text {pump }} / f_{\text {pump }}$, may be expanded to include multiple pulses, where $N_{\text {pump }}$ is the number of $\mathrm{THz}$ pulses included in the window. Taking the FFT of such a THz pulse train produces a series of comb teeth, with a tooth spacing of $f_{\text {pump }}$ and a transform limited FWHM (tooth width) of $f_{\text {pump }} / N_{\text {pump }}$. The comb teeth generated by processing a single $\mathrm{THz}$ pulse, as in standard implementations of ASOPS-THz-TDS, has a tooth width equal to the comb tooth spacing. This relation between the length of time delay sampled and tooth width allows an ASOPS-THz-TDS system to increase its effective resolution at the expense of its spectral coverage at a static $f_{\text {pump }}$.

Because scanning long time delay windows produces narrow comb teeth, THz-FCS with broadband $\mathrm{THz}$ spectral coverage is impossible at a static $f_{\text {pump }}$. Active control over the pump laser repetition rate is needed to interleave the comb teeth to perform broadband THz-FCS (since the carrier envelop phase is fixed). That is, it is necessary to vary $f_{\text {pump }}$ stepwise, taking data at each step, interleaving the comb teeth to cover the entire spectral region of interest. The first demonstration of broadband THz-FCS employed an Er:Fiber oscillator system with a comb tooth spacing of $25 \mathrm{MHz}$ and a tooth width of $2.5 \mathrm{MHz}$ was interleaved at $2.5 \mathrm{MHz}$ steps to partially resolve the rotational structure of a single K-stack of acetonitrile at 150 mTorr. ${ }^{23}$ The use of an Er:Fiber oscillator limits the $\mathrm{THz}$ bandwidth of this system, but its general principles of operation are similar to those of the system described here. Very narrow comb spacing and resolution via coherent multi-bunch $\mathrm{THz}$ emission from synchrotrons has recently been used to report similar measurements on acetonitrile ${ }^{24}$ up to a few hundred $\mathrm{GHz}$, but the broadband generation of $\mathrm{THz}$ radiation requiring a synchrotron source limits the potential for widespread spectroscopic applications and is not discussed further here.

\section{Summary of the system design and performance}

Motivated in large measure by the needs of high resolution $\mathrm{THz}$ spectroscopy, this manuscript presents the design and capabilities of a high-resolution, broadband ASOPS-THzTDS and THz-FCS system using two mode-locked $80 \mathrm{MHz}$ Ti:Sapphire femtosecond oscillators offset locked by a phaselocked loop (PLL) operating at the 60th harmonic $(4.8 \mathrm{GHz})$ of their repetition rate(s). Additionally, we synchronize the pump oscillator to a $\mathrm{Rb}$ frequency standard via a PLL operating at the 12th harmonic $(960 \mathrm{MHz})$ of the oscillator repetition rate. The outline of this paper is as follows. The capabilities of the new instrument are compared to selected ASOPS-THz-TDS and THz-FCS implementations in Table I. We describe the optics and briefly mention the PLL electronics employed by the instrument in Section II. For a full description of the PLL electronics, see the supplementary material. ${ }^{29}$ In Section III, we characterize the time delay sampling performance of the instrument using an air-gap etalon and an optical cross correlator.

In Section IV, gas-phase spectroscopy applications of the ASOPS-THz-TDS mode of operation are demonstrated by ASOPS-THz-TDS of $\mathrm{H}_{2} \mathrm{O}$ and acetonitrile. In particular, we measure line center frequencies of water vapor absorptions from 0.55 to $3.35 \mathrm{THz}$ and of $\mathrm{CH}_{3} \mathrm{CN}$ absorptions from 0.13 to $1.39 \mathrm{THz}$ in a short path length, low pressure cell. We obtain a signal-to-noise ratio (SNR) of $50 \mathrm{~dB}$ after $70 \mathrm{~min}$ of acquisition time. Comparisons of our measured values for water vapor to reference data yield a root-mean-square (RMS) frequency deviation of $14.7 \mathrm{MHz}$ and a mean frequency deviation of 11.6 MHz. While this ASOPS-THz-TDS mode covers a decade of bandwidth and is applicable to high-resolution gas-phase spectroscopy from 0.13 to $3.35 \mathrm{THz}$, the Dopplerlimited measurements demanded by remote sensing or in situ applications require even finer frequency resolution.

In Section V, we demonstrate the THz-FCS capabilities of the instrument. In THz-FCS mode, we have access to Dopplerlimited frequency resolution down to a RMS of $\sim 100 \mathrm{kHz}$ across the decade from 0.25 to $2.5 \mathrm{THz}$. Here, we use this

TABLE I. A summary of recently reported THz-TDS systems. Those demonstrated in frequency comb mode are listed below their parent systems. Detected bandwidth is defined as the envelope of the power spectrum above the noise floor in the frequency domain. Frequency resolution is defined as the comb tooth width or the repetition rate in ASOPS-THz-TDS systems. For THz-FCS systems, the step size employed to interleave comb teeth is given in parentheses next to the frequency resolution.

\begin{tabular}{|c|c|c|c|c|c|c|c|c|c|c|c|}
\hline $\begin{array}{l}\text { Oscillator } \\
\text { type }\end{array}$ & $\begin{array}{c}\text { Center } \\
\text { wavelength } \\
(\mathrm{nm})\end{array}$ & $\begin{array}{c}\text { Rep rate } \\
(\mathrm{MHz})\end{array}$ & $\begin{array}{l}\text { Pulse duration } \\
\text { (fs) }\end{array}$ & $\begin{array}{c}\text { Avg power } \\
(\mathrm{mW})\end{array}$ & $\begin{array}{c}\text { Detected } \\
\text { bandwidth } \\
(\mathrm{THz})\end{array}$ & $\begin{array}{c}\text { Offset lock } \\
(\mathrm{Hz})\end{array}$ & $\begin{array}{c}\text { Time delay } \\
\text { (ns) }\end{array}$ & $\begin{array}{c}\text { Frequency } \\
\text { resolution } \\
(\mathrm{MHz})\end{array}$ & $\begin{array}{l}\text { Averaging time } \\
(\mathrm{min})\end{array}$ & $\begin{array}{c}\text { Mean dev } \\
(\mathrm{MHz})\end{array}$ & Reference \\
\hline Ti:Sapph & 825 & 1000 & 45 & 800 & 7.0 & 2000 & 1.0 & 1000 & 1 & 142 & 18 and 19 \\
\hline Er:Fiber & 1550 & 56 & 50 & 110 & 1.5 & 5 & 17.8 & 56 & 16.7 & 4.0 & 20 \\
\hline Freq Comb & 1550 & 250 & 50 & 110 & 2.0 & 50 & 400 & $2.5(2.5)$ & 50 & 0.5 & 23 \\
\hline Ti:Sapph & 800 & 80 & $>100$ & 420 & 3.5 & 100 & 12.5 & 80 & 70 & 11.6 & This Work \\
\hline Freq Comb & 800 & 80 & $>100$ & 420 & 2.5 & 400 & 200 & $5(1.4)$ & 280 & 0.1 & 25, This Work \\
\hline
\end{tabular}


capability to fully resolve a K-stack of acetonitrile, which is not possible for the instrument in ASOPS-THz-TDS mode. We have previously demonstrated broadband THz-FCS with our Ti:Sapph based system by collecting data over an 18 pulse window to obtain a decade-spanning comb whose tooth spacing and width were $\sim 80 \mathrm{MHz}$ and $\sim 4.4 \mathrm{MHz}$, respectively, with precision tests provided by the measurement of Doppler limited linewidths for water rotational transitions at 10 mTorr. We also collected data over a 21470 pulse window to obtain a tooth width of $3.7 \mathrm{kHz} .{ }^{25}$ To conclude (Section VI), we outline near term upgrades to the system achievable via pulse compression and $\mathrm{THz}$ generation/detection optimization that would enable century-spanning bandwidth, that is, highresolution gas-phase spectroscopy from 0.1 to $10 \mathrm{THz}$.

\section{ASOPS-THz-TDS AND THz-FCS SYSTEM DESIGN}

We employ two Ti:Sapphire femtosecond oscillators (Micra-5, Coherent Inc.) factory equipped with cavity mirrors controlled by piezoelectric actuators (PZTs) enabling active control of their repetition rates. Both have repetition rates of $\sim 80 \mathrm{MHz}, \sim 420 \mathrm{~mW}$ of output power at a center wavelength of $\sim 800 \mathrm{~nm}$, and a pulse duration of $\sim 50 \mathrm{fs}$, which can be shortened to a transform-limited duration of $15 \mathrm{fs}$ with external pulse compression. The short pulse durations and high pulse bandwidths of these oscillators are ideal for pulsed $\mathrm{THz}$ generation and also enable broadband EO sampling of the generated $\mathrm{THz}$ pulses in the time-domain. The pump and probe oscillators are offset locked at $100 \mathrm{~Hz}$, covering $12.5 \mathrm{~ns}$ of time delay in $10 \mathrm{~ms}$ of scan time, supporting a theoretical maximum time-delay resolution of $15.6 \mathrm{fs}$.

The entire spectrometer is contained on a single floated optical table to isolate the system from low frequency acoustic noise and both the pump and probe lasers are chilled by the same water chiller to ensure that any temperature fluctuations are common mode. Other than these two considerations, no additional care is needed to operate the instrument as a frequency comb spectrometer. A schematic detailing the optical elements and the associated electronics employed by the system is shown in Figure 1. Due to the high bandwidth of the $800 \mathrm{~nm}$ pulses, it is advisable to use low group velocity dispersion (GVD) optics to minimize broadening of the pulses. As such, low GVD high-reflectivity mirrors (10B20UF.25, Newport) are employed throughout the system. Even so, external pulse compression is needed to mitigate the effects of GVD added by the chosen optics.

\section{A. THz generation}

The pump laser is used to generate pulses of $\mathrm{THz}$ radiation. A low GVD beam sampler (10B20-01NC.2, Newport) reflects $10 \%$ of the pump laser power, which is subsequently split by a series of two identical low GVD beam splitters (10RQ00UB.2, Newport) to provide power to the pump and clock photodiodes and the scan triggering subsystem, which are discussed later, and transmits $90 \%$ of the power. The transmitted power is focused through a low GVD plano-convex fused silica (FS) lens (SPX028, Newport) to a spot size of

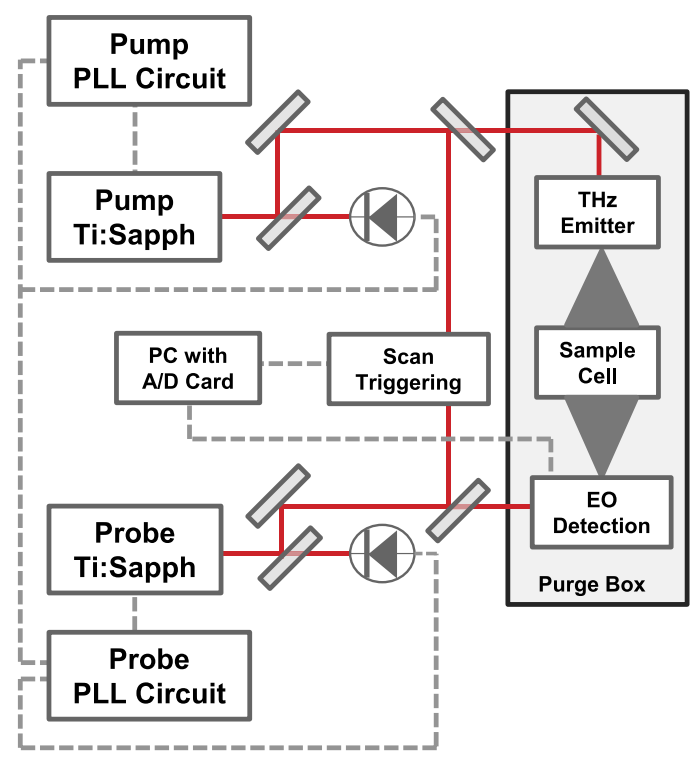

FIG. 1. A schematic of the ASOPS-THz-TDS system design. Solid red lines represent optical paths and dashed grey lines electrical signals. $\mathrm{THz}$ beam paths are represented by triangles.

$300 \mu \mathrm{m}$ to pump an interdigitated photoconductive $\mathrm{GaAs} \mathrm{THz}$ emitter (Tera-SED 3, Laser Quantum). ${ }^{26}$ Such large area emitters provide a substantial increase in average $\mathrm{THz}$ power and have bandwidth extending well into the 3-6 THz region when properly optimized. ${ }^{27}$ The emitter is DC biased at $15 \mathrm{~V}$. Each pump laser pulse incident on the emitter produces a pulse of $\mathrm{THz}$ radiation that is routed by off-axis parabolic mirrors (OAPMs) through a custom built sample cell with Topas (cyclic olefin copolymer) windows, an inner diameter of $5 \mathrm{~cm}$ and a path length of $12.7 \mathrm{~cm}$. Once $\mathrm{THz}$ pulses have interacted with the sample, they are focused onto a $1 \mathrm{~mm}$ thick, wedged ZnTe (110) crystal (INGCRYS Laser Systems Ltd.). A custom built purge box (Cleatech LLC) with an extra thick base and FS windows (210-1202E, Eksma Optics) encloses all of the THz routing optics and is flushed with dry nitrogen to remove water vapor from the $\mathrm{THz}$ beam path. $\mathrm{THz}$ pulses are subsequently detected via EO sampling by the $\mathrm{THz}$ detection subsystem.

\section{B. THz detection}

The probe laser is used to detect the pulses of THz radiation generated by the pump laser. A low GVD beam splitter (10RQ00UB.2, Newport) reflects half of the power from the probe laser to the $\mathrm{THz}$ detection subsystem and transmits half of the power to the scan triggering subsystem, discussed in Subsection II C. A low GVD beam sampler (BSF10-B, Thor Labs) subsequently reflects $10 \%$ of this transmitted power to the probe photodiode. The detection system senses THz pulses via the electro-optic effect. ${ }^{28}$ Probe pulses are sent through an indium-tin oxide (ITO) window, which is reflective at $\mathrm{THz}$ frequencies, allowing $800 \mathrm{~nm}$ probe pulses and $\mathrm{THz}$ pulses to be focused onto a $\mathrm{ZnTe}$ crystal.

Briefly, the $\mathrm{THz}$ pulses generate a transient birefringence in the ZnTe crystal, which rotates the polarization of the probe pulses by an amount directly proportional to the magnitude of the THz electric field. The probe pulses gate the EO 
detection, and so, their duration at the ZnTe crystal is critical. Thus, the use of low GVD optics along the probe beam path leading to the EO detection setup is essential. Once the $\mathrm{THz}$ and probe pulses have interacted, it is no longer important to avoid high dispersion optics. A doublet achromat (AC25475-B, Thor Labs) is used to focus the probe pulses through the polarization detection optics. The change in polarization induced by the $\mathrm{THz}$ field is measured by sending the probe pulse through a quarter waveplate $(\lambda / 4,10 \mathrm{RP} 54-2$, Newport), followed by a Wollaston prism (WP10-B, ThorLabs), which splits the probe pulse into its component polarizations. Finally, the THz-induced shift in polarization is detected by an ACcoupled balanced photodetector of $15 \mathrm{MHz}$ electrical bandwidth (PDB440A-AC, ThorLabs).

\section{Optical scan triggering}

An ASOPS based THz-TDS system requires a reliable scan triggering method to ensure accurate averaging of multiple scans. In our instrument, the scan triggering subsystem is essentially an optical cross correlator in which pump and probe pulses are focused off of the same OAPM onto a $\beta$-barium borate $(\mathrm{BBO})$ frequency doubling crystal. This configuration emits a pulse of $400 \mathrm{~nm}$ light each time a synchronized pumpprobe pulse pair arrives. An iris is used to block $400 \mathrm{~nm}$ light originating from asynchronized pulses and a band-pass filter (10BPF70-400, Newport) is used to block any residual $800 \mathrm{~nm}$ light from reaching a photomultiplier tube (PMT) (R928, Hamamatsu), which is part of an assembly that includes a built in high voltage power supply (H957-08, Hamamatsu). The PMT converts these $400 \mathrm{~nm}$ pulses into an electrical signal which is subsequently amplified using a current preamplifier (SR570, Stanford Research Systems). The amplified signal is used as the trigger for the digitizer card (ATS9462, AlazarTech) that records the time domain signal generated by the balanced photodetector. With the addition of optics described further in Section III, this subsystem can alternately be used as a time delay calibration etalon to characterize the time-delay accuracy of the instrument.

\section{Signal digitization and THz bandwidth}

Over the course of a full scan, the electric field of the THz waveform is sampled across the entire time delay between successive $\mathrm{THz}$ pulses. The analog signal from the balanced photodiodes is digitized by the 16-bit digitizer card (within a 32-bit averaging environment) at a user determined sampling rate and stored on a personal computer. The digitizer card sampling rate is referenced to a $10 \mathrm{MHz}$ Rb frequency standard (SIM 940, Stanford Research Systems) housed within a SIM 900 mainframe (Stanford Research Systems) to ensure an accurate time base, as any deviations in sampling rate will translate to errors in time delay. A frequency counter (5384A, HP) is also referenced to the Rb standard and monitors the pump laser repetition rate, which determines the precise amount of time delay covered during a single scan. This frequency counter also simultaneously monitors the probe laser repetition rate, which allows the user to verify that the PLL electronics are maintaining a constant offset.
We are able to use a balanced photodetector with a $3 \mathrm{~dB}$ point of $15 \mathrm{MHz}$, thanks to the time scaling effect of ASOPS. For a repetition rate of $80 \mathrm{MHz}$ and an offset lock of $100 \mathrm{~Hz}$, the time scaling factor is $1.25 \times 10^{-6}$ meaning that a maximum bandwidth of $12 \mathrm{THz}$ can be sampled with these settings. In Sections III and IV, we set the sampling rate to $160 \mathrm{MSa} / \mathrm{s}$ which gives two data points per probe pulse (incoming at a rate of $80 \mathrm{MHz}$ ). This sampling rate may then be decimated to lower rates such as $40 \mathrm{MSa} / \mathrm{s}$ in Section $\mathrm{V}$, where we operate at an offset lock of $400 \mathrm{~Hz}$ with a time scaling factor of $5 \times 10^{-6}$ sampling bandwidths of up to $3 \mathrm{THz}$.

As shown in Section V, we observe frequency content out to $3.5 \mathrm{THz}$, which is significantly less than the samplinglimited bandwidth of $12 \mathrm{THz}$. When substituting GaP for the $\mathrm{ZnTe}$ in the EO detection subsystem, we do not observe frequency content above $3.5 \mathrm{THz}$. For a GaAs THz emitter such as the one employed by our setup, bandwidth is highly dependent on the duration of the $800 \mathrm{~nm}$ pump pluses used. According to Drude-Lorentz simulations of GaAs interdigitated $\mathrm{THz}$ emitter behavior, a pulse width of $50 \mathrm{fs}$ will limit the emitter bandwidth to $12 \mathrm{THz}$ and a pulse width of $100 \mathrm{fs}$ will limit the emitter bandwidth to $6 \mathrm{THz} .{ }^{11}$ Since we do not employ a pulse compressor, it is likely that our pump pulse width is broadened to $>100 \mathrm{fs}$, leading to a lack of output above $3.5 \mathrm{THz}$. The covered bandwidth can therefore be improved in the future by compression of pump pulse width to below 50 fs to ensure emitter output up to $10 \mathrm{THz}$. This level of pulse compression is readily accomplished for our lasers, which have a transform limited pulse duration of $15 \mathrm{fs}$. We explore the timing jitter of the system in Section III, which is another potential factor that can limit the observed bandwidth.

\section{E. Phase-locked loop circuits}

Our ASOPS based THz-TDS employs a microwave frequency PLL circuit operating at the 60th harmonic of the respective laser repetition rates to maintain a constant frequency offset between the pump and probe lasers. The probe laser is equipped with two PZTs, one fast and one slow, which enable offset locking of the probe laser via slight adjustments of its cavity length by two separate cavity mirrors. To enable the use of our system as a frequency comb spectrometer, we additionally synchronize the pump laser repetition rate relative to a reference frequency via a second PLL operating at the 12th harmonic of the pump laser repetition rate. The pump laser is equipped with a slow PZT allowing active control of the pump laser repetition rate via slight displacement of a cavity mirror.

A full discussion of the PLL circuits along with a circuit diagram is provided in the supplementary material. ${ }^{29}$ Operating at high harmonics enables excellent phase discrimination and thus superior control. For example, in previous synchronization of two $100 \mathrm{MHz}$ Ti:Sapphire lasers across a long time delay, a precision of 4.3 fs was achieved with a PLL operating at the 80th harmonic. ${ }^{30}$ Here, the 60th harmonic was chosen as a balance between the performance of high bandwidth photodiodes and microwave circuitry and cost. Verification of the pump stabilization PLL circuit performance is readily accomplished via monitoring of the pump laser repetition rate by the frequency counter. Characterization of the time delay 
fidelity of the pump-probe offset lock is more involved, requiring measurement of precisely delayed laser pulses directly in the time-domain. We perform this measurement using an airspaced etalon, as described in Section III.

\section{CHARACTERIZATION OF INSTRUMENT TIME-DELAY PERFORMANCE}

The actual time-delay resolution of an ASOPS system is determined by the degree of accuracy to which the PLL electronics can maintain a constant repetition rate offset. ${ }^{16}$ The time delay axis is reset every time a new scan is triggered, but time-delay jitter can accumulate during each scan period. An additional concern is variable time-delay performance from scan to scan. To characterize these effects, we utilize a time delay calibration etalon; ${ }^{31}$ here, an air-spaced etalon formed by $90 \%$ reflective mirrors is inserted into the path of the pump laser before the cross correlator. ${ }^{32} \mathrm{~A}$ pair of quarter waveplates (10RP54-2, Newport) and a polarizing beam splitter cube (05FC16PB.5, Newport) serve to prevent back reflections from propagating toward to the pump laser.

This configuration transmits $1 \%$ of the pump laser power as a trigger pulse with no added time delay and $9 \%$ of the pump laser power as a series of pump pulses that have taken successively more round trips in the etalon cavity, with corresponding successive addition of time delay. The time delay added to a particular pump pulse by the etalon cavity $\tau_{\eta}$ is given by $\tau_{\eta}=\left(2 \times N \times l_{\eta}\right) / c$, where $N$ is the number of round trips taken by the pulse and $l_{\eta}$ is the distance between the inner faces of the etalon cavity mirrors. These pulses are detected by the triggering subsystem and digitized. The lasers are offset locked at $100 \mathrm{~Hz}$ during the measurement and so, the ultimate sensitivity of the following measurements is limited by the $2.2 \mathrm{~ns}$ rise time and $1.2 \mathrm{~ns}$ transit time jitter of the PMT, which are converted by the time scaling factor to $2.8 \mathrm{fs}$ and $1.5 \mathrm{fs}$, respectively.

A time-domain trace of the trigger signal acquired by averaging 10000 scans over 3.5 min of wall clock time is shown in Figure 2(a), the inset provides a zoomed in view to reveal signal out to 12 ns of delay. The peak centers and FWHM values for this averaged trace are fit using Gaussian line shapes. The average spacing between extracted peak center positions is calculated and used to determine that $\tau_{\eta}=794.089 \mathrm{ps}, l_{\eta}$
$=11.9031 \mathrm{~cm}$. This value of $\tau_{\eta}$ is used to generate a calibrated delay-time axis. The measured time delay, $\tau^{*}$, is extracted from each peak center and compared to the calibrated time-delay, $\tau$, yielding a RMS deviation of $1.4 \mathrm{fs}$ and a maximum deviation of 2.3 fs [see Figure 2(b)]. Since this is a co-added measurement, any systematic timing jitter would accumulate and shift the mean deviation away from zero, but random timing jitter will average out.

Random timing jitter that changes from scan-to-scan can be observed by measuring deviations in the FWHM of the peaks obtained. The RMS deviation is $3.7 \mathrm{fs}$ with a maximum deviation of 4.9 fs [Figure 2(c)]. Calibration of time delay using an air-spaced etalon is an attractive method for precise characterization of timing jitter, thanks to the simultaneous acquisition of all of the time delay peaks by a single measurement, and the accuracy of the fixed length of the etalon cavity. ${ }^{33}$ However, the optics associated with the air-spaced etalon and cross correlator, especially the polarizing beam splitter cube, add significant group delay dispersion (GDD, $>1000 \mathrm{fs}^{2}$ ). Consequently, the measured FWHM values of $600 \mathrm{fs}$ are not indicative of the actual laser pulse widths at the $\mathrm{THz}$ emitter and electro-optic detection crystal. The $1 \mathrm{MHz}$ bandwidth of the current preamplifier additionally limits the time delay resolution of the measured pulse widths. The actual jitter of the PLL circuit is likely near the detection threshold of these measurements.

To confirm this assumption, we conducted an additional measurement of the timing jitter of the system by determining the power level of the noise relative to the carrier signal of $4.87 \mathrm{GHz}$ at $+7 \mathrm{dBm}$ in the frequency domain. We characterized the frequency content and power level of the error signal of the final mixer using a baseband network analyzer (SR770, Stanford Research Systems). Since the bandwidth of the probe fast loop is $14 \mathrm{kHz}$ and scan triggering is at $100 \mathrm{~Hz}$, we integrated the noise contributions from $100 \mathrm{~Hz}$ to $14 \mathrm{kHz}$. This calculation estimates the random RMS jitter over the loop bandwidth to be 15.4 fs. Since the theoretical maximum time delay sampling of this instrument is $15 \mathrm{fs}$, a timing jitter at or near this level implies that the bandwidth of the instrument is not currently limited by the PLL electronics. These measurements may be verified via detection of improved bandwidth in the future, once improvements to the $\mathrm{THz}$ generation and detection subsystems have been implemented.
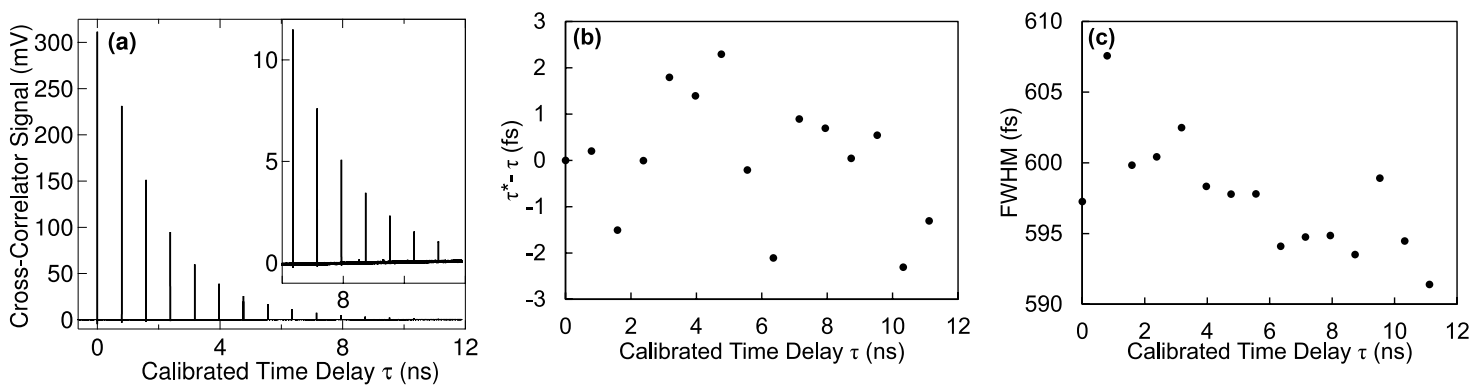

FIG. 2. (a) Raw time domain trace of successively delayed cross-correlator peaks. To obtain the calibrated time delay, the center of the first peak, which is used to trigger the scan, is set to zero. The inset details signal extending to $12 \mathrm{~ns}$. (b) Deviation of the measured minus calibrated time delay ( $\tau^{*}-\tau$ ) vs. calibrated time delay $(\tau)$. The RMS deviation is $1.4 \mathrm{fs}$ with a maximum deviation of $2.3 \mathrm{fs}$. (c) FWHM of etalon cross-correlation peaks vs. calibrated time delay. The RMS deviation is $3.7 \mathrm{fs}$ with a maximum deviation of $4.9 \mathrm{fs}$. 


\section{STATIC THz FREQUENCY COMB SPECTROSCOPY OR ASOPS-THz-TDS}

Here, the performance of our high-resolution ASOPS based THz-TDS system is characterized via the comparison of experimental water vapor and acetonitrile rotational transition energies to the values listed by the JPL database. Since the comb tooth width and spacing are equal for this measurement, no adjustment of $f_{\text {pump }}$ is required to cover the entire spectral range. The frequency of the pump laser is locked to $79.980833 \mathrm{MHz}$ to ensure that comb teeth remain static while acquiring these data. The sample cell is evacuated to a background pressure of 0.01 Torr using a vacuum pump (Edwards RV5) and then a background scan is acquired. To reduce the noise floor and improve bandwidth, 200000 traces have been averaged together, requiring $70 \mathrm{~min}$ of acquisition time. No features due to molecular absorptions are visible in the blank spectrum at this background pressure and averaging time. A few spurious peaks show up in the low frequency end of the spectrum due to etalons or other sources of interference, but they appear at consistent frequencies for a fixed laser offset and can be ignored. Once a background trace has been acquired, the cell is dosed with a flow of water vapor or acetonitrile at a constant pressure and a sample scan is acquired requiring an additional $70 \mathrm{~min}$ of acquisition time.
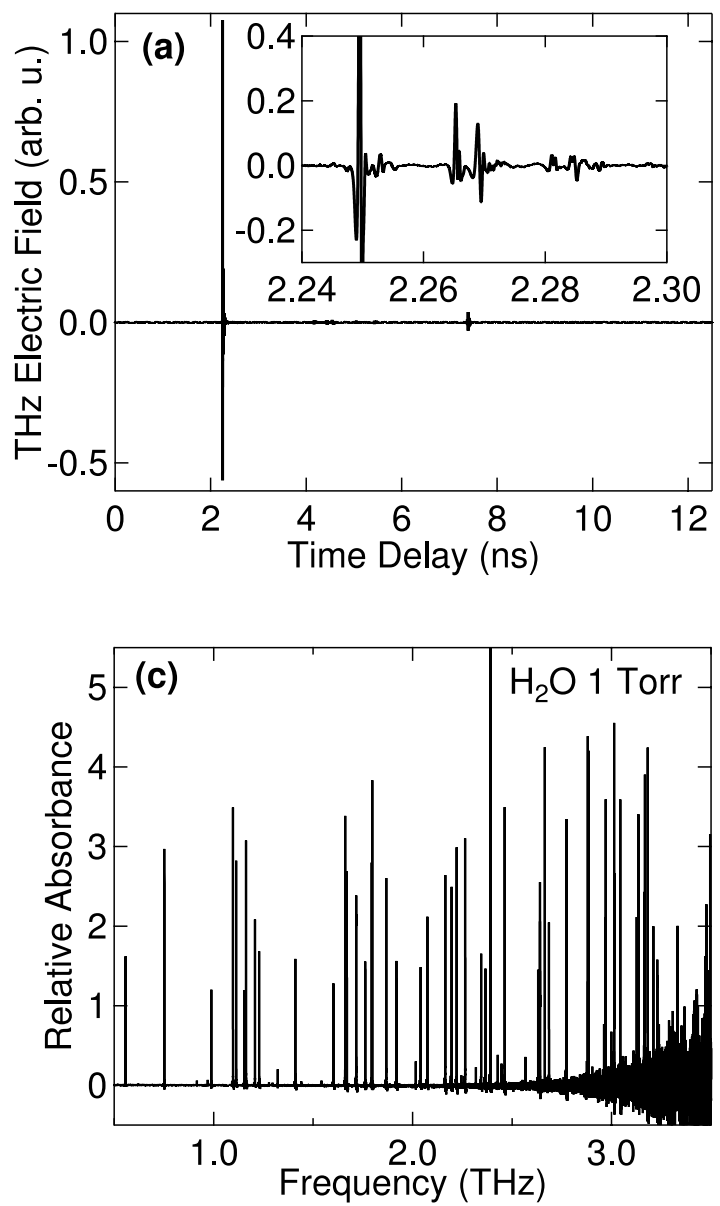

The raw data acquired provide a time-domain measurement of the $\mathrm{THz}$ electric field as a function of delay, as shown in Figure 3(a). The raw data are cut to a $5 \mathrm{~ns}$ window centered on the THz pulse and subsequent free induction decay to minimize noise contributions. Once windowed, the data are zeropadding to 8 additional powers of 2. Once a FFT is applied, zero-padding effectively interpolates the frequency-domain data. A power spectrum of the $\mathrm{THz}$ frequency content is then obtained by taking the FFT of the zero padded time-domain data. A power spectrum acquired after 70 min with 1 Torr of water vapor is shown in Figure 3(b). The SNR obtained after 70 min of acquisition is $50 \mathrm{~dB}$ measured from the peak of the power spectrum to the noise floor. Figure 3(c) shows a plot of relative absorbance obtained by taking the ratio of the relative power of the 1 Torr water vapor data shown in Figure 3(b) and the blank power spectrum obtained by taking a FFT of the blank time domain trace. This accounts for the variations in the power spectrum intrinsic to the instrument.

Once processed to obtain relative absorbance values, accurate values for line center frequencies are obtained with a simple peak picking algorithm. Relative absorbance data were acquired at water vapor pressures of 1 and 0.2 Torr as tests of the dynamic range of the system. To compare to $\mathrm{THz}$ spectral line catalogs, we calculated the sensitivity floor of the instrument in terms of the intensity units used by the JPL
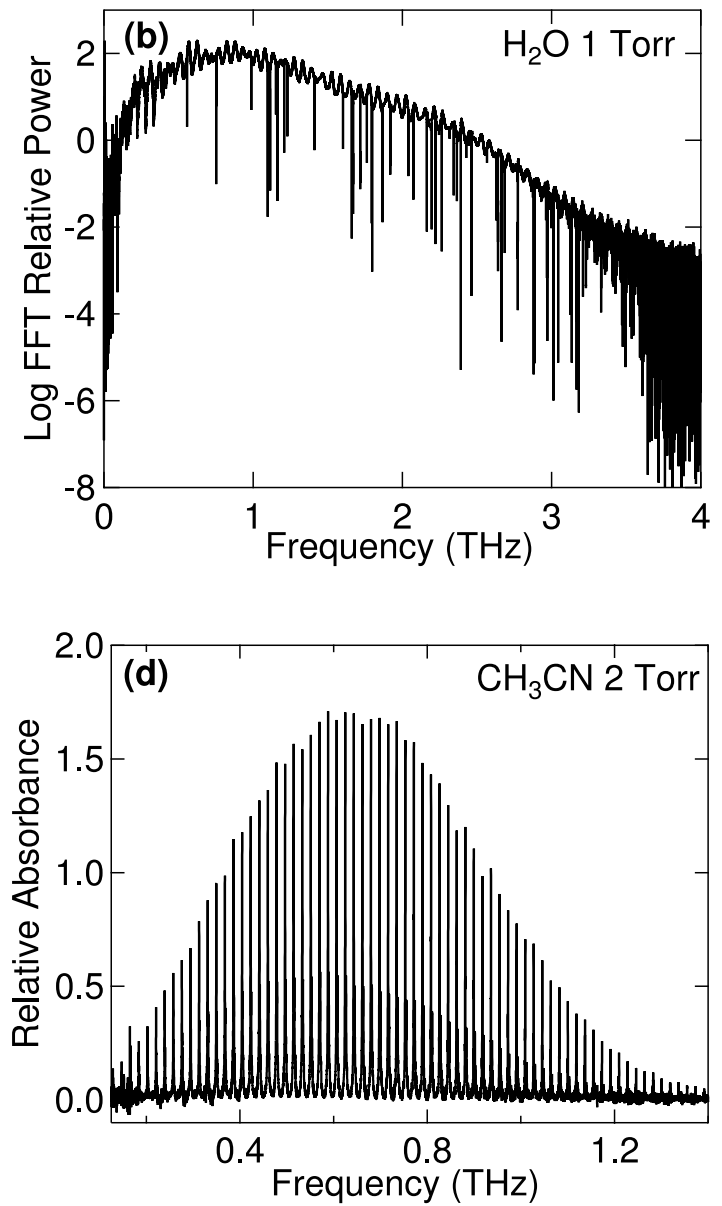

FIG. 3. THz time-domain trace acquired within $70 \mathrm{~min}$ at a background pressure of 0.01 Torr. Inset detail shows THz pulse electric field oscillations. (b) FFT relative power spectrum of a $\mathrm{THz}$ time-domain trace acquired within 70 min with $\mathrm{H}_{2} \mathrm{O}$ vapor at a pressure of 1 Torr. (c) Relative absorbance plot showing water vapor rotational transitions up to $3.35 \mathrm{THz}$. (d) Relative absorbance plot acquired at an acetonitrile $\left(\mathrm{CH}_{3} \mathrm{CN}\right)$ vapor pressure of 2 Torr showing rotational transitions down to $0.13 \mathrm{THz}$. 
database, namely, the base 10 logarithm of the integrated line intensity in units of $\mathrm{nm}^{2} \times \mathrm{MHz}^{34}$ Intense water absorption peaks for the 1 Torr data set begin to saturate, limiting the accuracy of this data set. A composite data set was therefore compiled using 34 lines with intensities between -2.68 and -0.80 from the 1 Torr data set and 32 lines with intensities above -0.80 from the 0.2 Torr data set. One line that was still saturated at 0.2 Torr was omitted.

By using the full suite of water measurements, we achieved a RMS deviation of $14.6 \mathrm{MHz}$ and a mean deviation of $11.6 \mathrm{MHz}$ in the composite data set acquired over $140 \mathrm{~min}$. For the individual spectral data sets, the mean frequency deviations were 14.9 and $23.3 \mathrm{MHz}$ at 0.2 and 1 Torr, respectively. Relative absorbance data for $\mathrm{CH}_{3} \mathrm{CN}(99.5 \%$ ACS grade, Mallinckrodt) at 2 Torr are shown in Figure 3(d). Acetonitrile does not have any saturated lines and provides an example of the ability of the instrument to accurately measure the relative intensity of transitions across a decade of bandwidth. Since the peak spacing of acetonitrile is as low as a few $\mathrm{MHz}$, we cannot resolve individual line structure in ASOPS-THz-TDS mode. This is the best reported resolution for an ASOPS based THz-TDS system over the covered bandwidth from 0.13 to $3.35 \mathrm{THz}$. In the future, the dynamic range performance of the instrument may be improved by increasing the length of the sample cell.

\section{INTERLEAVED THz FREQUENCY COMB SPECTROSCOPY}

THz-FCS is enabled by the high stability of the repetition rate of the pump laser, which ensures that time delay between adjacent $\mathrm{THz}$ pulses remains constant across long $\mathrm{THz}$ pulse trains. If the time axis is cut into a $12.5 \mathrm{~ns}$ windows and processed by a FFT, we obtain the ASOPS-THz-TDS data shown in Figure 3(b). If, however, multiple pulses are selected in a window that is an integer multiple of $12.5 \mathrm{~ns}$ and that entire window is processed by a FFT, then the frequency content from all of the pulses within the window is extracted. Since the tooth width is limited only by the number of $\mathrm{THz}$ pulses included in the FFT, the resolution of the instrument is readily adjustable in THz-FCS mode. Fundamentally, the only limitation to the resolution is the stability of the pump laser over the measurement time scale of interest.

However, there are additional experimental limitations imposed by the need to step the comb accurately to match the desired resolution of the comb tooth. The scanning time required to obtain a spectrum with a given SNR increases linearly with the number of frequency steps taken. Using a long pulse train to obtain narrow comb teeth leads to data processing and storage limitations imposed by the number of data points required to record a window including a large number of pulses. Fortunately, for Doppler-limited spectroscopy, the narrowest tooth width required is $\sim 100 \mathrm{kHz}$, which is yielded by the acquisition of $\sim 795$ consecutive THz pulses. Such pulse trains can be readily acquired by our system; however, they require custom user programming of the digitizer card. For Doppler-limited spectroscopy of acetonitrile, acquisition of 16 consecutive $\mathrm{THz}$ pulses provides a sufficient tooth width and this data can be recorded using the software included with the digitizer card. Here, we demonstrate the THz-FCS mode of our instrument by fully resolving the rotational structure of acetonitrile at 150 mTorr.

Acquisition of data in THz-FCS mode only requires minor changes to operating settings. No additional hardware is needed. We do not presently lock the pump laser repetition rate in FCS mode because the stability of the free running pump laser is sufficient over time periods of $\sim 5 \mathrm{~min}$ to achieve tooth widths of a few $\mathrm{MHz}$. We use a Proportional-IntegralDerivative (PID) controller (SIM960, Stanford Research Systems) to step the control voltage on the pump slow PZT in order to step the repetition rate of the pump laser. For more precise measurements requiring sub-MHz comb tooth widths, the pump laser repetition rate may be locked to the precisely desired value once the voltage step is applied.

For the measurements reported here, the output frequency of the probe DDS is set to $70.0240 \mathrm{MHz}$, with offset locking of the pump and probe lasers at $\Delta f=400 \mathrm{~Hz}$. The digitizer card is set to a sampling rate of $40 \mathrm{MS} / \mathrm{s}$, allowing the acquisition of $16 \mathrm{comb}$ teeth over the $40 \mathrm{~ms}$ window. The sample cell is dosed with acetonitrile at the desired pressure of 150 mTorr. For each scan, a total of 5000 traces are averaged together requiring $3.33 \mathrm{~min}$ of integration time, with an example of the resulting trace shown in Figure 4(a). After saving the averaged time domain trace, the control voltage on the pump slow PZT is increased by $175 \mathrm{mV}$ shifting the repetition rate of the pump laser down by $100 \mathrm{~Hz}$. A new scan is then run and saved at the new pump laser repetition rate. This process is repeated stepwise 58 times across the entire $-5 \mathrm{~V}$ to $+5 \mathrm{~V}$ range of the pump slow PZT, scanning the pump laser repetition rate across a frequency range of $5.8 \mathrm{kHz}$. Over the course of these scans, the comb teeth are interleaved at a step size of $1.4 \mathrm{MHz}$.

The time domain trace for each pump laser frequency step is FFT processed to yield a frequency comb with a tooth spacing of $80 \mathrm{MHz}$ and a tooth width of $5 \mathrm{MHz}$. A peak picking algorithm measures the intensity of each comb tooth and these values are stored. A 50 point boxcar average of the peak intensities is taken and these boxcar averaged values are used as the background spectrum since the majority of comb teeth in the spectrum are not attenuated by absorptions. This backgrounding method works best for the sparse spectra shown here and care should be taken in applying such an algorithm to denser spectra. The values obtained by the peak picking algorithm for all 58 frequency steps are compiled into an aggregate data set, such as that shown in Figure 4(b). The present THz-FCS implementation of the instrument only achieves $2.5 \mathrm{THz}$ bandwidth due to the limited detector bandwidth at $400 \mathrm{~Hz}$, but has an enhanced resolution of $5 \mathrm{MHz}$ across this entire bandwidth.

The background comb tooth intensities obtained by the boxcar average are then used to calculate a percent power absorbance value for each comb tooth, and these values are plotted in the percent power absorbance spectrum shown in Figure 4(c). We demonstrate the ability of the instrument to resolve Doppler-limited linewidths in THz-FCS mode using acetonitrile at a pressure of 150 mTorr in Figure 4(d). Each line shown in Figure 4(c) then breaks into the group of lines shown in Figure 4(d), now cleanly isolated at a spectral resolution of $5 \mathrm{MHz}$. It should be noted that the linewidth for 

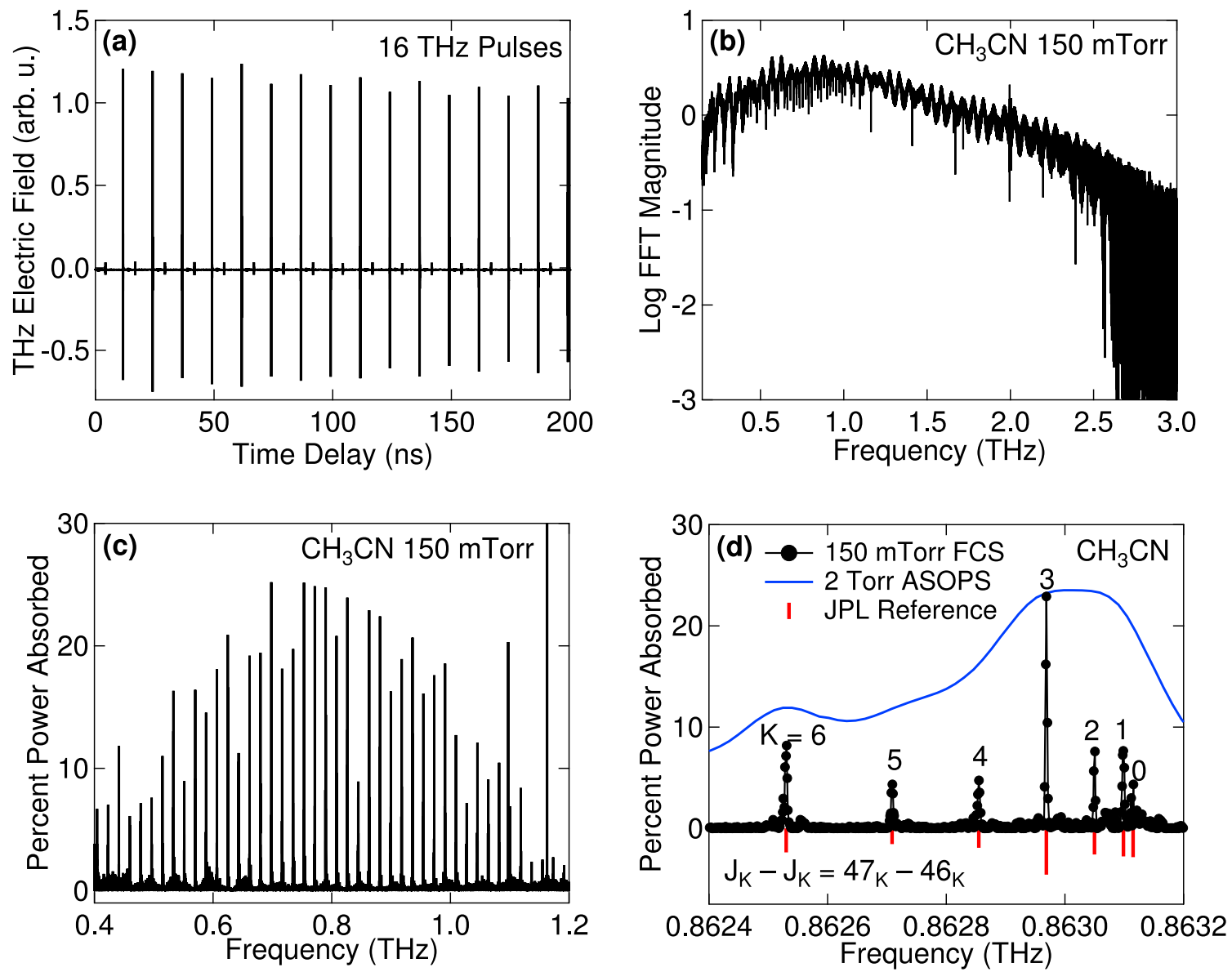

FIG. 4. (a) THz time-domain trace acquired within $3.33 \mathrm{~min}$ at an acetonitrile vapor pressure of $150 \mathrm{mTorr}$. (b) FFT relative power spectrum of all $58 \mathrm{THz}$ time-domain traces acquired with an acetonitrile $\left(\mathrm{CH}_{3} \mathrm{CN}\right)$ vapor pressure of $150 \mathrm{mTorr}$. (c) Percent power absorbance plot showing acetonitrile rotational transitions. (d) Detail of acetonitrile percent power absorbance plot showing the ability to resolve Doppler limited lines with $5 \mathrm{MHz}$ resolution. Data points acquired via peak picking raw data are plotted as black points. The comparable data from Figure 3(d) with $80 \mathrm{MHz}$ resolution are scaled to arbitrary units and plotted as a blue line highlighting that the instrument is only able to resolve these transitions in THz-FCS mode. Predicted line positions from the JPL reference catalog are plotted as vertical red lines with height scaled to the predicted linear absorbance intensity. Lines are labeled with their associated quantum numbers.

the ASOPS-THz-TDS trace is fundamentally limited by the instrument in this experiment and not by pressure broadening. The pressure of 2 Torr was chosen to maximize the number of lines resolved in the spectrum shown in Figure 3(d). As such, this instrument represents a nearly ideal compromise between fast-scanning systems with low resolution and slowscanning systems with high-resolution as the spectrometer can switch between ASOPS-THz-TDS mode and THz-FCS mode seamlessly.

\section{CONCLUSION}

We have constructed a high-resolution ASOPS-based time-domain spectrometer using two $\sim 80 \mathrm{MHz}$ repetition rate femtosecond Ti:Sapphire oscillators that can acquire both ASOPS-THz-TDS and THz-FCS data using the same hardware. In ASOPS-THz-TDS mode, the oscillators are offset locked at $100 \mathrm{~Hz}$, scanning a time delay of $12.5 \mathrm{~ns}$ in $10 \mathrm{~ms}$ of wall clock time. The pump oscillator is locked to a $\mathrm{Rb}$ frequency standard with $\mathrm{mHz}$ precision. We demonstrate the performance of ASOPS-THz-TDS by measuring rotational transitions of water vapor from 0.55 to $3.35 \mathrm{THz}$ with a RMS frequency deviation of $14.6 \mathrm{MHz}$. A decade spanning bandwidth has been demonstrated by the spectroscopy of acetonitrile down to $0.13 \mathrm{THz}$. Our current ASOPS-THz-TDS system thus supports gas-phase spectroscopy at a resolution of $80 \mathrm{MHz}$ from 0.13 to $3.35 \mathrm{THz}$, with excellent relative intensity capabilities (and the prospect for excellent absolute intensity measurements with accurate pressure control, for stable species).

In THz-FCS mode, the instrument generates a similarly broadband frequency comb, since the same $\mathrm{THz}$ emission and detection systems are employed. Here, we have demonstrated a tooth width of $5 \mathrm{MHz}$ with a variable tooth spacing of $79.9808 \mathrm{MHz} \pm 2.9 \mathrm{KHz}$. The unlocked pump oscillator in this case is stepped by $100 \mathrm{~Hz}$ increments across a range of $5.8 \mathrm{kHz}$ enabling the $\mathrm{THz}$ comb to fully resolve rotational transitions of gas phase molecules with Doppler-limited accuracy. An interleaved comb with $5 \mathrm{MHz}$ spectral resolution is sufficient to fully resolve a K-stack of rotational transitions of acetonitrile, but further reductions in tooth width are readily feasible should experimental needs dictate such, down to a few $\mathrm{kHz}$ if necessary with the existing instrumentation. Additionally, our demonstration of a broadband frequency comb highlights the dual functionality of the instrument using the same optical systems and digitization hardware and software, making this 
an ideal system to run survey scans in ASOPS-THz-TDS mode and then to generate high-quality reference spectra for molecules of interest with the THz-FCS mode.

Near term improvements to the spectrometer bandwidth will utilize dispersion compensation of the pump and probe lasers output to deliver 15 fs transform-limited pulses at the $\mathrm{THz}$ emitter and detector, thereby exploiting the theoretical maximum time delay sampling resolution of $15.6 \mathrm{fs}$ between successive probe pulses. This will increase the useful spectroscopic bandwidth of the instrument from $3.2 \mathrm{THz}$ to at least $7 \mathrm{THz} .{ }^{19}$ Further optimization of THz emission efficiency at high frequencies by implementing a reflection emitter geometry and detection by multiple inorganic crystals or poled EO sensitive polymers ${ }^{35}$ should enable gap-free measurement of gas phase signatures to at least $10 \mathrm{THz}$, covering nearly a century of bandwidth. ${ }^{11}$ Thus, this instrument overcomes many limitations of past THz-TDS and ASOPS-THz-TDS systems and demonstrates future paths to high precision, high dynamic range gas-phase spectroscopy covering the entire $\mathrm{THz}$ region.

\section{ACKNOWLEDGMENTS}

The authors are grateful to Jun Ye (NIST-JILA) for loaning equipment and for many helpful discussions. The authors thank M. A. Allodi (Caltech) for edits to this manuscript and for advice regarding alignment of optics and Jeff Groseth (Caltech) for advice and assistance with electronics. This work was supported by the National Science Foundation (Grant Nos. CHE-1214123 and CHE-0722330). I. A. Finneran acknowledges the NSF Graduate Research Fellowship Program for financial support.

${ }^{1}$ P. U. Jepsen, D. G. Cooke, and M. Koch, Laser Photonics Rev. 5, 124 (2011). ${ }^{2}$ M. Tani, R. Fukasawa, H. Abe, S. Matsuura, K. Sakai, and S. Nakashima, J. Appl. Phys. 83, 2473 (1998).

${ }^{3}$ R. Falconer and A. Markelz, J. Infrared, Millimeter, Terahertz Waves 33, 973 (2012).

${ }^{4}$ T. Q. Luong, P. K. Verma, R. K. Mitra, and M. Havenith, J. Phys. Chem. A 115, 14462 (2011).

${ }^{5}$ H. Harde, R. A. Cheville, and D. Grischkowsky, J. Phys. Chem. A 101, 3646 (1997).

${ }^{6}$ W. D. Langer, T. Velusamy, J. Pineda, P. Goldsmith, D. Li, and H. W. Yorke, "Galactic observations of terahertz $\mathrm{c}^{+}\left(\right.$got c $\left.^{+}\right)$: Cii detection of warm 'dark gas' in the ISM," in Conditions and Impact of Star Formation: New Results with Herschel and Beyond, EAS Publications Series Vol. 52, edited by M. Rollig, R. Simon, V. Ossenkopf, and J. Stutzki (EDP Sciences, Cedex A, 2011), pp. 161-164.

${ }^{7}$ T. G. Phillips and J. Keene, Proc. IEEE 80, 1662 (1992).
${ }^{8}$ M. A. Allodi, S. Ioppolo, M. J. Kelley, B. A. McGuire, and G. A. Blake, Phys. Chem. Chem. Phys. 16, 3442 (2014).

${ }^{9}$ S. Ioppolo, B. A. McGuire, M. A. Allodi, and G. A. Blake, Faraday Discuss. 168, 461 (2014).

${ }^{10} \mathrm{D}$. Mittleman, in Sensing with Terahertz Radiation, edited by D. Mittleman (Springer-Verlag, Berlin, 2002), p. 337, ISBN: 3540431101

${ }^{11}$ P. J. Hale, J. Madeo, C. Chin, S. S. Dhillon, J. Mangeney, J. Tignon, and K. M. Dani, Opt. Express 22, 26358 (2014).

${ }^{12}$ B. J. Drouin, F. W. Maiwald, and J. C. Pearson, Rev. Sci. Instrum. 76, 10 (2005).

${ }^{13}$ S. Bartalini, L. Consolino, P. Cancio, P. De Natale, P. Bartolini, A. Taschin, M. De Pas, H. Beere, D. Ritchie, M. S. Vitiello, and R. Torre, Phys. Rev. X 4, 7 (2014).

${ }^{14}$ M. Rosch, G. Scalari, M. Beck, and J. Faist, Nat. Photonics 9, 42 (2015).

${ }^{15}$ P. A. Elzinga, R. J. Kneisler, F. E. Lytle, Y. Jiang, G. B. King, and N. M. Laurendeau, Appl. Opt. 26, 4303 (1987).

${ }^{16}$ A. Bartels, R. Cerna, C. Kistner, A. Thoma, F. Hudert, C. Janke, and T. Dekorsy, Rev. Sci. Instrum. 78, 8 (2007).

${ }^{17}$ J. Johnson, J. A. Johnson, S. Grubel, S. Johnson, S. Graebel, A. Ferrer, and T. Feurer, J. Opt. Soc. Am. B 31, 1035 (2014).

${ }^{18}$ G. Klatt, R. Gebs, C. Janke, T. Dekorsy, and A. Bartels, Opt. Express 17, 22847 (2009).

${ }^{19}$ G. Klatt, R. Gebs, H. Schaefer, M. Nagel, C. Janke, A. Bartels, and T. Dekorsy, IEEE J. Sel. Top. Quantum Electron. 17, 159 (2011).

${ }^{20}$ T. Yasui, K. Kawamoto, Y. D. Hsieh, Y. Sakaguchi, M. Jewariya, H. Inaba, K. Minoshima, F. Hindle, and T. Araki, Opt. Express 20, 15071 (2012).

${ }^{21}$ S. A. Diddams, D. J. Jones, J. Ye, S. T. Cundiff, J. L. Hall, J. K. Ranka, R. S. Windeler, R. Holzwarth, T. Udem, and T. W. Hänsch, Phys. Rev. Lett. 84, $5102(2000)$

${ }^{22}$ T. Udem, R. Holzwarth, and T. W. Hänsch, Nature 416, 233 (2002).

${ }^{23}$ Y. D. Hsieh, Y. Iyonaga, Y. Sakaguchi, S. Yokoyama, H. Inaba, K. Minoshima, F. Hindle, T. Araki, and T. Yasui, Sci. Rep. 4, 7 (2014).

${ }^{24}$ S. Tammaro, O. Pirali, P. Roy, J.-F. Lampin, G. Ducournau, A. Cuisset, F. Hindle, and G. Mouret, Nat. Commun. 6, 7733 (2015).

${ }^{25}$ I. A. Finneran, J. T. Good, D. B. Holland, P. B. Carroll, M. A. Allodi, and G. A. Blake, Phys. Rev. Lett. 114, 5 (2015).

${ }^{26}$ A. Dreyhaupt, S. Winnerl, M. Helm, and T. Dekorsy, Opt. Lett. 31, 1546 (2006).

${ }^{27}$ S. Matsuura, G. A. Blake, R. A. Wyss, J. C. Pearson, C. Kadow, A. W. Jackson, and A. C. Gossard, Appl. Phys. Lett. 74, 2872 (1999).

${ }^{28}$ P. C. M. Planken, H. K. Nienhuys, H. J. Bakker, and T. Wenckebach, J. Opt. Soc. Am. B 18, 313 (2001).

${ }^{29}$ See supplementary material at http://dx.doi.org/10.1063/1.4932567 for details regarding PLL circuit design and operation.

${ }^{30}$ L. S. Ma, R. K. Shelton, H. C. Kapteyn, M. M. Murnane, and J. Ye, Phys. Rev. A 64, 021802 (2001).

${ }^{31}$ G. Sucha, M. Fermann, D. Harter, and M. Hofer, IEEE J. Sel. Topics Quantum Electron. 2, 605 (1996).

${ }^{32} \mathrm{D}$. Holland, "Design, construction and applications of a high resolution terahertz time-domain ASOPS spectrometer," Ph.D. thesis, Caltech, 2014.

${ }^{33}$ M. Kinoshita, H. Iida, and Y. Shimada, IEEE Trans. Terahertz Sci. Technol. 4, 756 (2014)

${ }^{34}$ H. M. Pickett, R. L. Poynter, E. A. Cohen, M. L. Delitsky, and J. C. Pearson, J. Quant. Spectrosc. Radiat. Transfer 60, 883 (1998).

${ }^{35}$ P. D. Cunningham, N. N. Valdes, F. A. Vallejo, L. M. Hayden, B. Polishak, X.-H. Zhou, J. Luo, A. K.-Y. Jen, J. C. Williams, and R. J. Twieg, J. Appl. Phys. 109, 043505 (2011). 